\title{
OLYMPUS
}

Your Vision, Our Future

\section{Bioluminescence microscopy for cellular level circadian analysis in the suprachiasmatic nucleus}

\author{
Traditionally, luminescence has not been able to provide sufficient spatial resolution at the \\ microscopic level without the use of expensive imaging equipment. The new Olympus LV200 \\ microscope has changed all this; its unique optical geometry and precise incubation capabilities \\ enable acquisition of even the faintest signals with cellular-level detail. This has opened up new \\ frontiers in many areas of research, especially in the study of circadian rhythm-related events.
}

The Olympus LV200 microscope (Fig. 1) has enabled cellular-level analysis of a structure in the hypothalamus of the mammalian brain, called the suprachiasmatic nucleus (SCN). The SCN contains a 'daily clock' that generates near-24-hour (circadian) rhythmic variations in both physiology and behavior. This clock is synchronized to changes in environmental illumination (day-night variations) by light information, which is conveyed directly to the SCN by a specialized input from the eye. The SCN neurons send clock information to the rest of the brain and body via nerve pathways and secretion of particular chemicals. Some SCN cells contain the necessary molecular apparatus to function as single-cell timekeepers ('clock cells'), whereas others lack this property. The ability to identify clock cells and the chemicals via which they communicate with one another as well as with the rest of the brain is key to research in this field. Fluorescence microscopy is not always effective for such studies because repeated exposure to the illuminating radiation can cause rapid deterioration in the health of tissues. Luminescence is an alternative that has not traditionally provided the high spatial resolution required, but the advances made in the Olympus LV200 challenge this limit.

\section{The model of a modern bioluminescence microscope} The Olympus LV200 Luminoview has been optimized for collecting the faint light associated with bioluminescence, providing consistent cellular-level clarity. The path from the object to the camera is straight and as short as possible to ensure that as much light as possible reaches the charge-coupled device (CCD) chip. Therefore, there are no unnecessary additional mirrors, filters or lenses, which can all contribute to light absorption. What is more, the tube lens has an extremely high numerical aperture (NA), which affords a vast increase

\section{Werner Kammerloher}

Olympus Life Science Europa GmbH, Microscopy, Wendenstrasse 14-18, D-20097 Hamburg, Germany. Correspondence should be addressed to W.K (microscopy@olympus-europa.com).

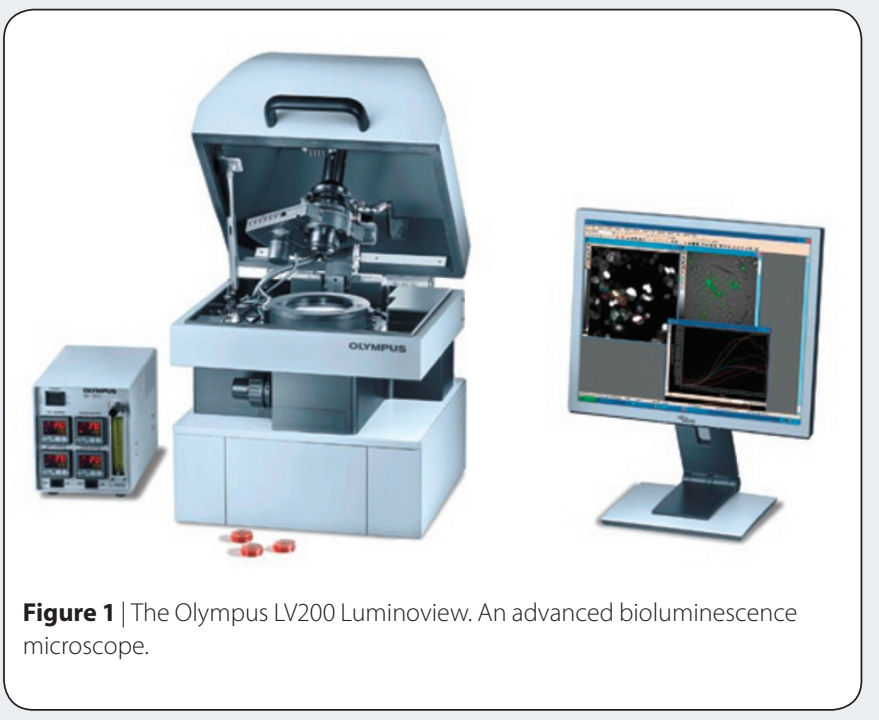

in sensitivity when compared to conventional microscope optics. This enables the Olympus LV200 to produce signal outputs many times higher than traditional systems and therefore to use conventional CCD or electron-multiplying CCD cameras rather than expensive liquid nitrogen-cooled devices. These unique optical properties allow exquisite single-cell resolution not previously possible with standard imaging equipment.

\section{Environmentally aware}

With the optical components optimized for the detection of luminescent light, the LV200 is housed inside a light-tight box that also serves as a precisely controllable environmental chamber. The system provides independent temperature controls for the stage, incubation chamber, top cover and objective to ensure that temperature is maintained as precisely as possible. This also ensures that the major components are all at the same temperature, avoiding expansion or contraction issues and condensation on the objective. A water reservoir 
APPLICATION NOTES

provides the correct humidity level, ensuring that the components of the growth medium remain at the correct concentration. $\mathrm{CO}_{2}$ flow control enables $\mathrm{pH}$ stability, and culture medium can be replenished or changed using a special pump system.

Such environmental control enables samples to be continuously monitored over days or even weeks, without the need to move the sample. The optical path has also been designed to enable dualcolor luminescence as well as transmitted-light fluorescence imaging. Furthermore, with standard brightfield illumination and phase contrast inserts, target areas of the sample can be found easily before switching to luminescence detection. It is therefore also possible to produce luminescence and fluorescence overlays on brightfield images, which provides users with the capability to localize and colocalize proteins more precisely.

\section{The advantages in practice}

Hugh Piggins, Alun Hughes and Clare Guilding at the University of Manchester's Faculty of Life Science are looking at the long-term expression of the protein Period-2 (PER2) in mice, a protein encoded by the Per2 gene, a key 'clock' gene. They have been using the Olympus LV200 to look at long-term expression patterns of the PER2 protein, a process that requires acutely cultured brain slices to be incubated and imaged for extended periods of time. Recording images once every 3 minutes for up to 7 days, they can then analyze the gross expression of PER2 over these times within the entire culture, as well as view each individual cell to look for variations from and similarities to the gross expression (Fig. 2).

\section{Conclusions}

Luminescence has been overshadowed by fluorescence for many years, mostly because of the expensive image collection systems required. The advantages that luminescence offers over fluorescence

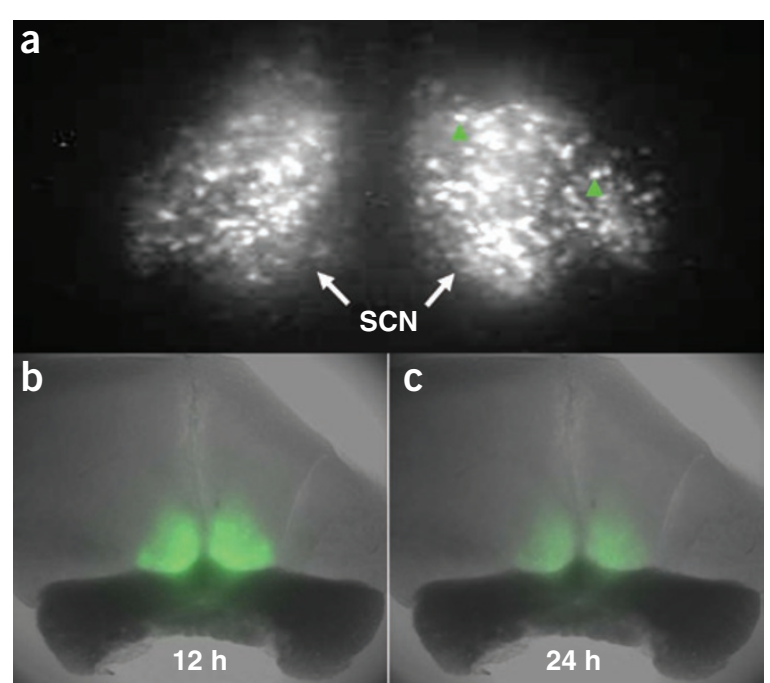

Figure 2 | Micrograph images captured on the LV200, showing both its cellular and tissue-level resolution capabilities. (a) High-magnification image of the bilateral SCN, with individual cells highlighted by green arrowheads. (b,c) PER2::LUC luciferase fusion protein expression (green) superimposed on a light transmission image of the SCN culture. Note that the levels of PER2::LUC bioluminescence show large changes across the 24-h day (high at $12 \mathrm{~h}$, lower at $24 \mathrm{~h}$ ). Images courtesy of H. Piggins.

for long-term live cell imaging, though, make it very attractive to researchers. The LV200 system represents a sea change in luminescence imaging capabilities, as its optics have been optimized for luminescence.

This article was submitted to Nature Methods by a commercial organization and has not been peer reviewed. Nature Methods takes no responsibility for the accuracy or otherwise of the information provided. 